\title{
Evaluating the Sensitivity of (miR-378) as a Circulatory Screening Biomarker for Diabetic Cardiomyopathy: Comparative Study
}

\author{
Sana Abdul-Jabbar Ali ${ }^{1}$, Hyder Hashim Al-Shlah ${ }^{2}$, Mais Mazin Al-Hamdani ${ }^{3}$, Oday Jasim Al-salihi ${ }^{4}$ \\ ${ }^{1}$ Assistant Lecturer, Al-Safwa University, Iraq, ${ }^{2,4}$ Prof., Faculty of medicine/Babylon University/Iraq, \\ ${ }^{3}$ Assistant Lecturer, Faculty of pharmacy/Al-Ayen University/Iraq
}

\begin{abstract}
Background: Diabetic cardiomyopathy is one of the serious complication of diabetes mellitus, with a silent development and it is often underestimated, Currently available diagnostic techniques are limited in their ability to identify patients who present with uncommon symptoms or silent myocardiopathy in its early stages.
\end{abstract}

Aim: To evaluate role of miRNA-378 compared to GLS as a sensitive biomarker for early detection of subclinical diabetic cardiomyopathy.

Method: The echocardiographic assessment for GLS was done for control group (H) and normotensive diabetic patients and these patients were divided into (CD) group without cardiomyopathy, and (M)group with cardiomyopathy diagnosed by negative GLS and positive GLS, respectively .Group (F) include Hypertensive and/or ischemic diabetic patients with overt heart failure.

Results: There is significant elevation in miR-378 Ct value between $\mathrm{H}$ in one side \& other three diabetic gps. $(\mathrm{CD}, \mathrm{M}, \mathrm{F})$ as presented in table $(3-17)(\mathrm{p}<0.05)$. There is significant elevation in miR-378 Ct value in $\mathrm{M}$ than CD groups. There is significant elevation in $\mathrm{Ct}$ value in $\mathrm{F}$ than $\mathrm{M}$ gp. $(\mathrm{p}=0.000)$. In the ROC curve (AUC) was 0.965 . The sensitivity and specificity were 0.978 and 0.646 , respectively according to the chosen cut-off value.

Conclusion: This study showed that miRNA-378 could be regarded as a novel sensitive biomarker which could play a significant role for early detection of subclinical diabetic cardiomyopathy.

Keywords: Diabetic cardiomyopathy, screening, GLS, sensitivity, miRNA-378.

\section{Introduction}

Diabetic cardiomyopathy was first described by Rubler in $1972^{[1,2]}$. The term describes several mechanisms involved in the pathogenesis of this entity including changes inmyocardial structure and metabolism that are not directly attributed to other co-morbidities

\section{Corresponding Author:}

Sana Abdul-Jabbar Ali

Assistant Lecturer, Al-Safwa University, Iraq

e-mail: saagzz2012@yahoo.com such as coronary artery disease (CAD) or hypertension. Left ventricular hypertrophy, myocardial lipotoxicity, increased oxidative stress, cell death from apoptosis, impaired contractile reserve, altered substrate utilization as free fatty acids (FFA), mitochondrial dysfunction and fibrosis are among the mechanisms involved with these changes in structure and metabolism contributing to the progression of heart failure ${ }^{[2]}$. Cardiac magnetic resonance (CMR) is the gold standard in the assessment of myocardial fibrosis given that $\mathrm{T} 1$ sequences separate normal from fibrotic tissue ${ }^{[3]}$. Nonetheless, CMR is not widely available, and it is time and cost consuming with longer and exhausting protocols. Speckle tracking echocardiography has been validated against magnetic 
resonance imaging ${ }^{[4,5]}$. Global longitudinal strain (GLS) is the simplest deformation parameter specified by Speckle tracking echocardiography and probably the closest to routine clinical application ${ }^{[6]}$. Quantitative assessment of myocardial function is now possible with this technique ${ }^{[7]}$. Routine use of Speckle tracking echocardiography in daily clinical practice is not costeffective in this scenario and currently impractical for large-scale population screening. Therefore, assessment of individual risk factors becomes very important in clinical practice especially in primary care settings.More than half of patients with heart failure have preserved ejection fraction $(\mathrm{HFpEF})^{[8]}$. In several studies, BNP proved to be a suboptimal screening test to detect preclinical LV dysfunction or $\mathrm{LVH}^{[9-11]}$. For all these reasons novel sensitive markers are needed. Although many substances have been suggested as biomarkers for $\mathrm{DbCM}$, none have qualified for clinical use. Diagnosis and therapies are difficult because of a lack of specific biomarkers and imaging techniques. There is a need to anticipate the progression of heart failure in patients with diabetes and for that reason we propose a strategy of non-invasive analysis of inflammation-fibrosis LVD. The detection of appropriate biomarkers could potentially permit routine population-wide screening, allowing early diagnosis and anticipation of cardiac dysfunction, and stratify these subclinical cases ${ }^{[12,13]}$.
MicroRNAs (miRNAs) are short noncoding RNAs that modify gene expression by regulating mRNA stability or translation during various disease processes. Each mRNA regulates expression of multiple genes. Dysregulated miRNAs are potentially involved in the pathogenesis of DbCM ${ }^{[14]}$. There are multiple miRNAs that have been implicated with the progression of disease. After release from the cell, these molecules remain stable in extracellular fluids and can be measured by quantitative polymerase chain reaction $(\mathrm{qPCR})^{[15]}$.Clinical studies have proposed peripheral blood microRNAs (miRNAs) as sensitive, specific and non-invasive biomarkers for the early monitoring of alterations in cardiac viability, structure, and function. Distinctive circulating miRNA signatures with clinical value have been described in various cardiomyopathies and type 2 diabetes and its related vascular complications ${ }^{[16,17]}$.

\section{Subjects, Materials and Method}

Subjects: From May 2018 to April 2019, a total of 75 patients with DM2 and 25 control subjects who were aged 40-65 yr were prospectively enrolled from Diabetic Center, CCU, and Echo Unit in Merjan Medical City (M.M.C.) admitted as diagnosed cases of DM2 by expert physicians. Each 25 patients group has a definite characters as in table (1).

Table (1): Diagnostic criteria of study groups

\begin{tabular}{|l|l|}
\hline CD group & Normotensive diabetic patients without cardiomyopathy diagnosed by negative strain echo study \\
\hline M group & Normotensive diabetic patients with cardiomyopathy diagnosed by positive strain echo study \\
\hline F group & Hypertensive and/or ischemic diabetic patients with heart failure diagnosed by conventional echo study \\
\hline H group & Apparently healthy subjects with negative strain echo study \\
\hline
\end{tabular}

All were subjected to clinical examination including, heart rate and blood pressure. Further clinical details are shown in Supplementary Table (2).

Table (2): Clinical data for patients in all study groups and control subjects

\begin{tabular}{|c|c|c|c|c|c|}
\hline \multirow{2}{*}{\multicolumn{2}{|c|}{ Variables }} & \multicolumn{4}{|c|}{ Groups } \\
\hline & & CD Group & M Group & F Group & H Group \\
\hline \multicolumn{2}{|l|}{ Total number (n) } & 25 & 25 & 25 & 25 \\
\hline \multicolumn{2}{|l|}{ Age/mean $\pm \mathrm{SD}$} & $52.76 \pm 6.3$ & $53.04 \pm 6.2$ & $58.44 \pm 5.9$ & $50.88 \pm 8.1$ \\
\hline \multirow{2}{*}{ Gender } & Male & 14 & 15 & 18 & 17 \\
\hline & Female & 11 & 10 & 7 & 8 \\
\hline \multicolumn{2}{|l|}{$\mathrm{BMI}\left(\mathrm{Kg} / \mathrm{m}^{2}\right) /$ mean $\pm \mathrm{SD}$} & $26.4 \pm 4.1$ & $32.1 \pm 5.3$ & $30.6 \pm 3.5$ & $29.2 \pm 5.9$ \\
\hline \multirow{2}{*}{ Family history of DM2 } & $+\mathrm{ve}$ & 19 & 17 & 18 & 4 \\
\hline & -ve & 6 & 8 & 7 & 21 \\
\hline \multicolumn{2}{|l|}{$\mathrm{HbA} 1 \mathrm{c} \%$} & $8.5 \pm 2.3$ & $8.9 \pm 2.3$ & $9.1 \pm 2.2$ & $4.4 \pm 0.27$ \\
\hline
\end{tabular}




\section{Method}

Echocardiography: The gold standard biomarker of diagnosis was GLS\% which was defined as the average longitudinal strain at end-systole in 18 segments. By regarding cut-off value of GLS $=-18 \%$ according to (Islam E. Shehata et al ${ }^{[18]}$ who reported that a GLS cutoff of $<-18.1 \%$ was able to accurately "predict subclinical LV systolic dysfunction".

Strain echocardiography: Speckle tracking strain mode was selected on the echo-machine during apical 4, 2 and 3-chamber imaging and three consecutive cycles were recorded at a frame rate of 60 to 80 frame/sec. The LV is divided into 6 walls (inferoseptum, lateral, anterior, inferior, posterior and anteroseptal walls) every wall is divided into basal, mid and apical segments except the anteroseptum and posterior wall divided into basal and mid segments only, the global PSLS value for each participant were calculated as the average of values of the 16 segments ${ }^{[18]}$.

Blood collection and Laboratory method: Venous blood was acquired by venipuncture without anticoagulant treatment. Samples were centrifuged and subsequently aliquoted and stored at $-80^{\circ} \mathrm{C}$ prior to analysis.For RNA isolation, an miRNeasy Mini Kit (Sigma/USA) was used to extract total RNA from 500 $\mu 1$ of serum. Reverse transcription (RT) was performed using a TaqMan MicroRNA Reverse Transcription Kit (Sigma/USA). The RT product was preamplified, and level of miR-378-5p (assay MIRAP00354) were measured by qPCR using a TaqMan miRNA assay (Applied Biosystems).miRNA species with CT value $\geq 35$ were considered below the detection threshold. The serum miRNA expression levels in individual samples were determined by a TaqMan probe-based RT-qPCR on a 7300 Real-Time PCR Sequence Detection System (Applied Biosystems). Because U6 and 5S rRNA are degraded in serum samples and the lack of a consensus housekeeping miRNA for the RT-qPCR analysis of serum miRNAs, miRNA expression was normalized to serum volume ${ }^{[19]}$. For serum and exosome samples. Influence of haemolysis was discarded by analysing the $\mathrm{Ct}$ values of miR-23a and miR-451a $\mathrm{a}^{[20]}$.

\section{Results and Discussion}

The difference in miRNA-378 circulating level in different study groups: There is significant elevation in the $\mathrm{Ct}$ value of miR-378 with the progression of the diabetic cardiomyopathy as presented in table (4)
Medico-legal Update, January-March 2021, Vol. 21, No. 1

1303

indicating its down regulation as the disease progress. This result agreed with (Rui Guo et al) who classify miR378 as anti-hypertrophic miRNAs as it is downregulated with the development of $\mathrm{DbCM}^{[21]}$.

This result disagreed with (Sarah Costantino,) who stated that miR-378 is one of key initiators of apoptosis, were significantly over expressed in the diabetic heart and intensive glycemic control was unable to revert these changes ${ }^{[22]}$. The complex biology of miRNAs may also influence the respective findings. The miRNA profile of a given cell is highly specific to the stressor which it is exposed and suggests that miRNA expression in the human heart is dynamically regulated as a function of the pathophysiological context ${ }^{[23]}$.

There is significant elevation in miR-378 Ct value between $\mathrm{H} \&$ other three diabetic gps. (CD, M, F) as presented in table (5) $\mathrm{p}<0.05$; which represent its relevance to DM2. This result agreed with (Ivana Knezevic et al) who reported that in tissues such as fibroblasts and fetal hearts, where insulin-like growth factor receptor-1(IGF1R) levels are high, we found either absent or significantly low miR-378 levels, suggesting an inverse relationship between these two factors [24]. IGF-R are hybrid receptors which are more abundant when insulin receptors are down-regulated in response to the hyperinsulinemia as seen in insulin-resistant patients with DM2 as stated by Liam J. Murphy ${ }^{[25]}$ Also it agreed with (Assmann et $\mathrm{a}^{[26]}$ ) who stated that it is down regulated with DM2. miR-378 down regulation relevance to DM2 approved by (Yong Zhang et $\mathrm{al}^{[27]}$ ) when they found that $\mathrm{Tg}$ mice had a significant overall increase in body $\mathrm{O}_{2}$ consumption, $\mathrm{CO}_{2}$ production, and energy expenditure.

The significant elevation in miR-378 Ct value in $M$ than $C D$ groups indicate its downregulation with the development of cardiomyopathy in its subclinical state as that miR-378 downregulated in case of hypertrophy development and oxidative stress increase ${ }^{[28]}$. These findings confirm the potential role of these modulators in identifying $\mathrm{DbCM}$ in relevance to their role in mitochondrial metabolism of cardiomyocyte via FoxO1 as presented by (Puthanveetil, P. $^{[29]}$ ) who stated that overexpression of FoxO1 mimics a hyperglycemic effect on vascular endothelium and also that hyperglycemiainduced endothelial dysfunction is mediated through FoxO1. miR-378 mimics reduced Akt phosphorylation also resulted in decreased phosphorylation of FoxO1, ultimately leading to FoxO1 activation. This effect was 
also perceived in a $\mathrm{H} 2 \mathrm{O} 2$-induced oxidative stress model in rat cardiomyocytes. So downregulation of miR-378 resulted in increased expression of FoxO1 which resulted in mitochondrial dysfunction trigger cardiac cell death in diabetic heart disease.

There is significant elevation in $\mathrm{Ct}$ value in $\mathrm{F}$ than $\mathrm{M}$ gp. $(\mathrm{p}=0.000)$ which indicate more downregulation of miR-378 in F gp. than $\mathrm{M}$ gp.as presented in table (5). This lower expression may be due to different pathophysiological background related to hypertension $\&$ ischemia as concluded by (Jan Fiedler et $\mathrm{al}^{[30]}$ ) who published that presence of miR-378 attenuated ischemiainduced apoptosis by inhibiting caspase- 3 expression in cardiac myocytes and blunted cardiac hypertrophy and dysfunction upon cardiac overload by targeting Ras signaling. So may be the ischemic hypertensive metabolic changes resulted in downregulation of miR378.

Table (4): ANOVA-All Groups with Ct of miR-378

\begin{tabular}{|c|c|c|c|c|}
\hline \multicolumn{5}{|c|}{ Descriptive } \\
\hline \multicolumn{5}{|c|}{ Ct of miR-378 } \\
\hline \multirow{2}{*}{ Groups } & \multirow{2}{*}{ Mean } & \multirow{2}{*}{ Std. Deviation \pm} & \multicolumn{2}{c|}{$\mathbf{9 5 \%}$ Confidence Interval for Mean } \\
\cline { 3 - 5 } & & & Lower Bound & Upper Bound \\
\hline CD & 19.6 & 2.1 & 18.80208 & 20.54192 \\
\hline F & 30.4 & 3.4 & 28.97827 & 31.89006 \\
\hline H & 17.6 & 2.9 & 16.30735 & 18.89526 \\
\hline M & 25.9 & 3.4 & 24.39589 & 27.45138 \\
\hline
\end{tabular}

Table (5): Comparison between groups

\begin{tabular}{|c|c|c|c|c|c|}
\hline \multicolumn{6}{|c|}{ Multiple Comparisons } \\
\hline \multicolumn{6}{|c|}{ Dependent Variable: Ct of miR-378 } \\
\hline \multicolumn{6}{|c|}{ LSD } \\
\hline \multirow{2}{*}{\multicolumn{2}{|c|}{ Groups }} & \multirow{2}{*}{ Mean Difference } & \multirow{2}{*}{ p-value } & \multicolumn{2}{|c|}{ 95\% Confidence Interval } \\
\hline & & & & Lower Bound & Upper Bound \\
\hline \multirow{3}{*}{$\mathrm{H}$} & $\mathrm{CD}$ & -2.070 & .020 & $-3.80996-$ & $-.33143-$ \\
\hline & $\mathrm{F}$ & -12.832 & .000 & $-14.58941-$ & $-11.07632-$ \\
\hline & M & -8.322 & .000 & $-10.11752-$ & $-6.52714-$ \\
\hline \multirow{2}{*}{ M } & $\mathrm{CD}$ & 6.251 & .000 & 4.49190 & 8.01137 \\
\hline & $\mathrm{F}$ & $-4.510_{-*}^{*}$ & .000 & $-6.28734-$ & $-2.73372-$ \\
\hline
\end{tabular}

miRNA-378 as a screening marker: To evaluate the potential diagnostic value of miRNA-378, the ROC curve has been used. For cut-off value, values exceeding the cut-off are positive, while those below the cut-off are negative.

It is found that the area under the ROC curve (AUC) was 0.965 (Fig. 1). The sensitivity and specificity were
0.978 and 0.646 , respectively. The cut-off $\mathrm{Ct}$ value was 20.06, it was appeared to have independent associations with the clinical end-points that are studied. As a bloodbased marker, the miR-378 characteristics as highly sensitive may improve the screening values of DbCM. These results suggested that miR-378 may be highly sensitive and moderately specific enough to detect DbCM. 


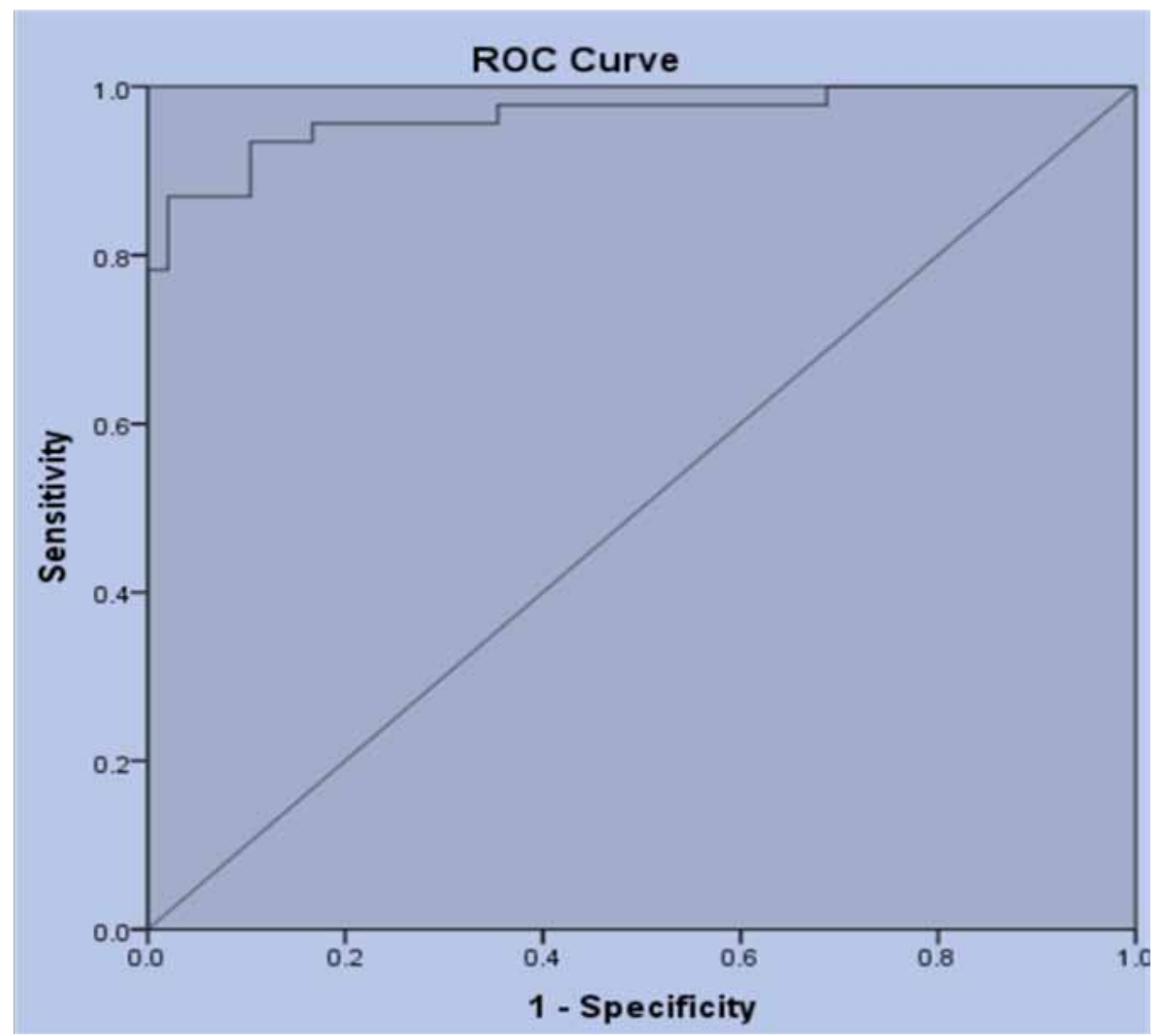

Figure (1): ROC curves for miRNA-378 Ct value in all study groups

The extremely high sensitivity (0.978) of miR-378 to identify $\mathrm{DbCM}$ patients from non-symptomatic diabetic individuals exceeded our most optimistic expectations.

These findings strengthen the clinical applicability of circulating miR-378 as a biomarker of diabetic cardiomyopathy in type 2 diabetes patients.

\section{Conclusion}

Serum miR-378 expression level can be regarded as a non-invasive tool to improve the detection, prediction, and monitoring of cardiac-related complications in the early stages of diabetes.

Ethical Clearance: The Research Ethical Committee at scientific research by ethical approval of both $\mathrm{MOH}$ and MOHSER in Iraq

\section{Conflict of Interest: None}

Funding: Self-funding

\section{References}

1. Rubler S, Dlugash J, Yuceoglu YZ, et al. New type of cardiomyopathy associated with diabetic glomerulosclerosis. Am J Cardiol. 1972;30:595602.

2. Boudina S, Abel ED. Diabetic cardiomyopathy, causes and effects. Rev Endocr Metab Disord. 2010;11:31-9.

3. Saeed M, Van TA, Krug R, et al. Cardiac MR imaging: current status and future direction. Cardiovasc Diagn Ther. 2015;5:290-310.

4. Amundsen BH, Helle-Valle T, Edvardsen T, et al. Noninvasive myocardial strain measurement by speckle tracking echocardiography: validation against sonomicrometry and tagged magnetic resonance imaging. J Am Coll Cardiol. 2006;47: 789-93.

5. Saito M, Okayama H, Yoshii T, et al. Clinical significance of global two-dimensional strain as a surrogate parameter of myocardial fibrosis 
and cardiac events in patients with hypertrophic cardiomyopathy. Eur Heart J Cardiovasc Imaging. 2012;13:617-23.

6. Yingchoncharoen T, Agarwal S, Popovic ZB, et al. Normal ranges of left ventricular strain: a metaanalysis. J Am Soc Echocardiogr. 2013;26:185-91.

7. Mor-Avi V, Lang RM, Badano LP, et al. Current and evolving echocardiographic techniques for the quantitative evaluation of cardiac mechanics: ASE/EAE consensus statement on methodology and indications endorsed by the Japanese Society of Echocardiography. Eur $\mathrm{J}$ Echocardiogr. 2011;12:167-205.

8. Hogg K, Swedberg K, McMurray J. Heart failure with preserved left ventricular systolic function; epidemiology, clinical characteristics, and prognosis. J Am Coll Cardiol. 2004;43:317---27.

9. Fang ZY, Schull-Meade R, Leano R, Mottram PM, Prins JB, Marwick TH. Screening for heart disease in diabetic subjects. Am Heart J 2005 Feb 1;149(2):349-354.

10. Redfield MM, Rodeheffer RJ, Jacobsen SJ, Mahoney DW, Bailey KR, Burnett JC. Plasma brain natriuretic peptide to detect preclinical ventricular systolic or diastolic dysfunction. A communitybased study. Circulation 2004;109:3176-3181.

11. Kiencke S, Handschin R, von Dahlen R, et al. Pre-clinical diabetic cardiomyopathy: prevalence, screening, and outcome. Eur J Heart Fail 2010 Sep;12(9):951-957.

12. Devereux RB, Roman MJ, Paranicas M, et al. Impact of diabetes on cardiac structure and function: the Strong Heart Study. Circulation 2000;101:22712276.

13. Swoboda PP, McDiarmid AK, Erhayiem B, et al. Diabetes mellitus, microalbuminuria, and subclinical cardiac disease: identification and monitoring of individuals at risk of heart failure. $\mathrm{J}$ Am Heart Assoc 2017 Jul 1;6(7), e005539.

14. Quiat D, Olson EN. MicroRNAs in cardiovascular disease: from pathogenesis to prevention and treatment. J Clin Invest 2013 Jan;123(1):11-18

15. de Gonzalo-Calvo, D., Cenarro, A., Civeira, F. \& Llorente-Cortes, V. microRNA expression profile in human coronary smooth muscle cell-derived microparticles is a source of biomarkers. Clin Investig Arterioscler. (2016).28, 167-177
16. Ortega, F. J. et al. Profiling of circulating microRNAs reveals common microRNAs linked to type 2 diabetes that change with insulin sensitization. Diabetes Care. (2014).37, 1375-1383

17. Guo R, Nair S. Role of microRNA in diabetic cardiomyopathy: from mechanism to intervention. Biochim Biophys acta (BBA)-molecular basis Dis. 2017;1863(8):2070-7.

18. Shehata IE, Eldamanhory AS, Shaker A. Early predictors of left ventricular dysfunction in hypertensive patients: comparative cross-section study. Int J Cardiovasc Imaging. 2020;1-10.

19. Wang J, Pei Y, Zhong Y, Jiang S, Shao J, Gong J. Altered Serum MicroRNAs as Novel Diagnostic Biomarkers for Atypical Coronary Artery Disease. 2014;9(9): 17-22

20. Blondal, T. et al. Assessing sample and miRNA profile quality in serum and plasma or other biofluids. Method.(2013). 59, S1-6

21. Guo R, Nair S. Role of microRNA in diabetic cardiomyopathy: from mechanism to intervention. Biochim Biophys acta (BBA)-molecular basis Dis. 2017;1863(8):2070-7.

22. Costantino S, Paneni F, Lüscher TF, Cosentino F. MicroRNA profiling unveils hyperglycaemic memory in the diabetic heart. Eur Heart J. 2015;37(6):572-6.

23. Waldenström, A., Genneback, N., Hellman, U. \& Ronquist, G. Cardiomyocyte microvesicles contain DNA/RNA and convey biological messages to target cells. PLoS One. (2012).7, e34653

24. Knezevic I, Patel A, Sundaresan NR, Gupta MP, Solaro RJ, Nagalingam RS, et al. A novel cardiomyocyte-enriched MicroRNA, miR-378, targets insulin-like growth factor 1 receptor implications in postnatal cardiac remodeling and cell survival. J Biol Chem. 2012;287(16):1291326.

25. Liam J. Murphy, Insulin-Like Growth Factor-I: A Treatment for Type 2 Diabetes Revisited, Endocrinology, Volume 147, Issue 6, 1 June 2006, Pages 2616-2618

26. Assmann TS, Recamonde-Mendoza M, Costa AR, Puñales M, Tschiedel B, Canani LH, et al. Circulating miRNAs in diabetic kidney disease: case-control study and in silico analyses. Acta Diabetol. 2019;56(1):55-65. 
27. Zhang Y, Li C, Li H, Song Y, Zhao Y, Zhai L, et al. miR-378 activates the pyruvate-PEP futile cycle and enhances lipolysis to ameliorate obesity in mice. EBioMedicine. 2016;5:93-104.

28. Nagalingam RS, Sundaresan NR, Gupta MP, Geenen DL, Solaro RJ, Gupta M. A cardiacenriched microRNA, miR-378, blocks cardiac hypertrophy by targeting Ras signaling. J Biol Chem. 2013;288(16):11216-32.
29. Puthanveetil P. FoxO1-miRNA interacting networks as potential targets for mitochondrial diseases. Drug Discov Today. 2019;24(1):342-9.

30. Fiedler J, Baker AH, Dimmeler S, Heymans S, Mayr M, Thum T. Update on non-coding RNAs in vascular disease-from basic discoveries to clinical applications. Cardiovasc Res. 2018; 